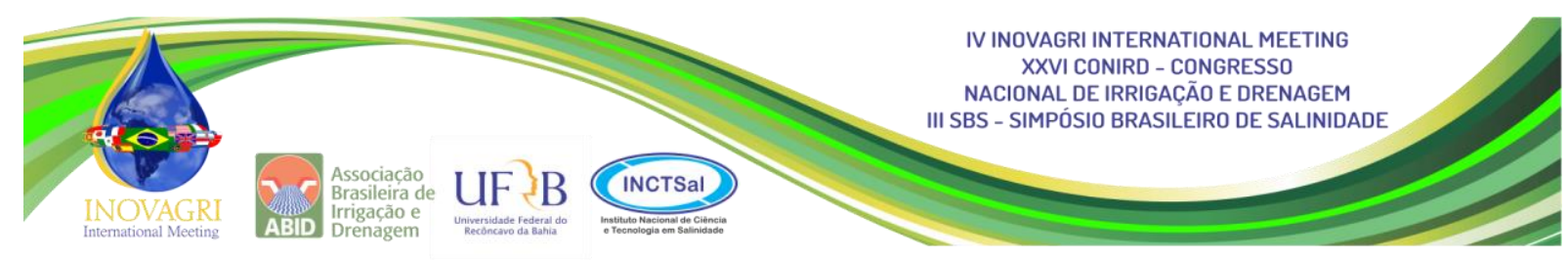

\title{
DESENVOLVIMENTO VEGETATIVO E TENSÃO DA ÁGUA NO SOLO EM VIDEIRAS PARA SUCO SOBRE DOIS PORTA-ENXERTOS
}

\author{
M. A. F. Conceição ${ }^{1}$, M. A. Tecchio ${ }^{2}$, M. A. Barbaráa ${ }^{3}$ J. R. de Souza ${ }^{3}$, \\ M. J. R. da Silva ${ }^{4}$, M. F. Moura ${ }^{5}$
}

RESUMO: O presente trabalho teve por objetivo avaliar o efeito de dois porta-enxertos sobre o desenvolvimento vegetativo e as tensões da água no solo em videiras destinadas à elaboração de suco, sob condições irrigadas. As avaliações foram realizadas de agosto a dezembro de 2015 no município de Votuporanga (SP), com a cultivar Isabel Precoce enxertada sobre os portaenxertos 'IAC 572' e 'IAC 766'. As plantas foram sustentadas no sistema espaldeira e irrigadas por microaspersores. Tensiômetros foram instalados a $0,10 \mathrm{~m}$ e 0,30 $\mathrm{m}$ de profundidade, para monitorar as tensões da água no solo. Foram determinados os índices de área foliar, a altura da copa e a porcentagem da área sombreada das videiras. As tensões da água no solo apresentaram comportamento semelhante nos dois porta-enxertos. Não foram observadas diferenças significativas entre as variáveis de crescimento vegetativo nas videiras. Concluiu-se que os porta-enxertos 'IAC 572' e 'IAC 766' não afetaram o desenvolvimento vegetativo e as tensões da água no solo para a cultivar Isabel Precoce, em condições irrigadas.

PALAVRAS-CHAVE: viticultura, irrigação, evapotranspiração

\section{VEGETATIVE DEVELOPMENT AND SOIL WATER TENSION IN JUICE GRAPEVINES ON TWO ROOTSTOCKS}

\footnotetext{
ABSTRACT: The objective of this study was to evaluate the effect of two rootstocks on the vegetative development and soil water tension for juice grapevines under irrigated conditions. The evaluations were carried out from August to December 2015 in Votuporanga (SP) using

${ }^{1}$ Pesquisador, Embrapa Uva e Vinho, Estação Experimental de Viticultura Tropical (EVT), Caixa Postal 241, CEP 15700-971, Jales, SP, marco.conceicao@embrapa.br

${ }^{2}$ Professor Doutor, Universidade Estadual Paulista (UNESP), Faculdade de Ciências agronômicas (FCA), Departamento de Horticultura, Botucatu, SP, tecchio@fca.unesp.br

3 Mestranda, Instituto Agronômico de Campinas, Centro de Frutas, Jundiaí, SP, marianebarbara@hotmail.com.br; julianarocha_agrofer@hotmail.com

${ }^{4}$ Doutorando, Universidade Estadual Paulista (UNESP), Faculdade de Ciências agronômicas (FCA), Departamento de Horticultura, Botucatu, SP, marlonjocimar@gmail.com

${ }^{5}$ Pesquisadora Científica, Instituto Agronômico de Campinas, Centro de Frutas, Jundiaí, SP, mouram@iac.sp.gov.br
} 
the cultivar Isabel Precoce grafted on 'IAC 572' and 'IAC 766' rootstocks. The plants were trained in a vertical shoot positioning system and irrigated by microsprinklers. Tensiometers were installed at $0.10 \mathrm{~m}$ and $0.30 \mathrm{~m}$ depth to monitor soil water tension. Leaf area index, plant height and percentage of shaded area of vines were determined. Soil water tension presented similar behavior in both rootstocks. No significant differences were observed between the vegetative growth variables in the vines. It was concluded that the rootstocks 'IAC 572' and 'IAC 766' did not affect the vegetative development and soil water tension for Isabel Precoce cultivar under irrigated conditions.

KEYWORDS: viticulture, irrigation, evapotranspiration

\section{INTRODUÇÃO}

A produção de uvas para elaboração de suco tem sido incrementada nas regiões tropicais do Brasil, sendo a 'Isabel Precoce' (Vitis labrusca) uma das principais cultivares empregadas com esse propósito (Camargo et al., 2011; Ribeiro et al., 2012). Dentre os porta-enxertos mais recomendados nessas regiões destacam-se o 'IAC 766' e o 'IAC 572' (Camargo et al., 2008).

Em condições irrigadas, o uso de diferentes porta-enxertos pode afetar a demanda hídrica da cultura em decorrência, principalmente, de alterações proporcionadas no vigor da copa (Edwards et al., 2014). Entre essas alterações, as que mais afetam a evapotranspiração da cultura são a altura da copa, o índice de área foliar e, principalmente, a porcentagem da área de solo sombreada pelas plantas (Allen \& Pereira, 2009). Williams \& Ayars (2005) demonstraram que videiras podem apresentar acréscimos na demanda hídrica se a percentagem da área sombreada pela copa (PAS) aumentar, mesmo mantendo-se constantes a área foliar e a altura da copa. Por essa razão, valores da PAS têm sido empregados, muitas vezes, na estimativa dos coeficientes de cultivo (Kc) de videiras (Hellman, 2016; Moyer et al., 2015).

Por outro lado, um aumento na demanda hídrica das videiras, em função de um maior vigor da copa, irá afetar a disponibilidade de água no solo para as plantas. Essa disponibilidade, por sua vez, poderá ser monitorada empregando-se sensores de umidade do solo instalados na região radicular da cultura (Nolz et al., 2016).

O presente trabalho teve, assim, o objetivo de avaliar o efeito dos porta-enxertos 'IAC 572' e 'IAC 766' sobre o desenvolvimento vegetativo e as tensões da água no solo na cv. Isabel Precoce, destinada à elaboração de suco de uva, sob condições irrigadas. 


\section{MATERIAL E MÉTODOS}

As avaliações foram realizadas de agosto a dezembro de 2015 em uma área experimental do Centro de Seringueira e Sistemas Agroflorestais do Instituto Agronômico de Campinas (IAC), localizado no município de Votuporanga (SP), noroeste do estado de São Paulo, situado a $20^{\circ} 20^{\prime} \mathrm{S}, 4^{\circ} 58^{\prime} \mathrm{O}$ e $510 \mathrm{~m}$ de altitude. De acordo com a classificação de Köppen, o clima local é classificado como tropical úmido (Aw), com temperatura média anual de $22,8^{\circ} \mathrm{C}$ e precipitação anual de $1.437 \mathrm{~mm}$ concentrada, principalmente, entre outubro e março. O solo é classificado como Argissolo Vermelho-Amarelo eutrófico, de textura média a arenosa (Alem et al., 2015).

A cultivar utilizada foi a 'Isabel Precoce' enxertada sobre os porta-enxertos 'IAC 572' e 'IAC 766'. As videiras de dois anos de idade foram conduzidas na direção norte-sul e sustentadas em um sistema do tipo espaldeira, com espaçamento de 2,0 m entre fileiras e 1,0 m entre plantas. O vinhedo foi coberto com tela de polietileno com $18 \%$ de sombreamento, para proteção contra o ataque de pássaros e morcegos. As avaliações foram realizadas em cinco plantas para cada porta-enxerto, em delineamento experimental inteiramente casualizado. Para as irrigações, foram empregados microaspersores com vazão média de $32 \mathrm{~L} \mathrm{~h}^{-1}$, espaçamento de 3,0 $\mathrm{m}$ x 2,0 m, suspensos na espaldeira e operando de forma invertida.

A tensão da água no solo foi determinada ao longo do ciclo da cultura empregando-se tensiômetros de punção. Os tensiômetros foram instalados nas fileiras de plantas a profundidades de $0,10 \mathrm{~m}$ e $0,30 \mathrm{~m}$, representando as camadas de solo de 0 a 0,20 m e de 0,20 m a 0,40 m, respectivamente. Essas camadas concentram a maior parte do sistema radicular da cultura (Conceição, 2015).

No final do ciclo da cultura, foram medidas as áreas foliares (AF) das videiras empregando-se metodologia não destrutiva apresentada por Regina et al. (2000). Nas mesmas plantas, foram determinadas a altura da copa (h) e a porcentagem da área sombreada (PAS). Os índices de área foliar (IAF) foram calculados dividindo-se os valores de AF pela área ocupada pelas plantas $\left(2,0 \mathrm{~m}^{2}\right)$. O valor de PAS foi obtido dividindo-se a largura da projeção da sombra da copa sobre o solo ao meio-dia pelo espaçamento entre fileiras (Hellman, 2016; Moyer et al., 2015):

$$
\mathrm{PAS}=100 . \mathrm{LS} / \mathrm{EF}
$$

Em que,

PAS - porcentagem da área sombreada pela copa (\%); 
LS - largura média da projeção da sombra da copa ao meio-dia (m);

$\mathrm{EF}$ - espaçamento entre fileiras (m).

A largura projeção da copa foi determinada mediante a utilização de um dispositivo graduado adaptado conforme recomendação de Hellmann (2016), medindo-se o sombreamento a partir da linha de plantio (Figura 1).

A comparação entre os valores médios de IAF, h e PAS, para os dois porta-enxertos, foi realizada empregando-se o teste $\mathrm{F}$ ao nível de $5 \%$ de probabilidade.

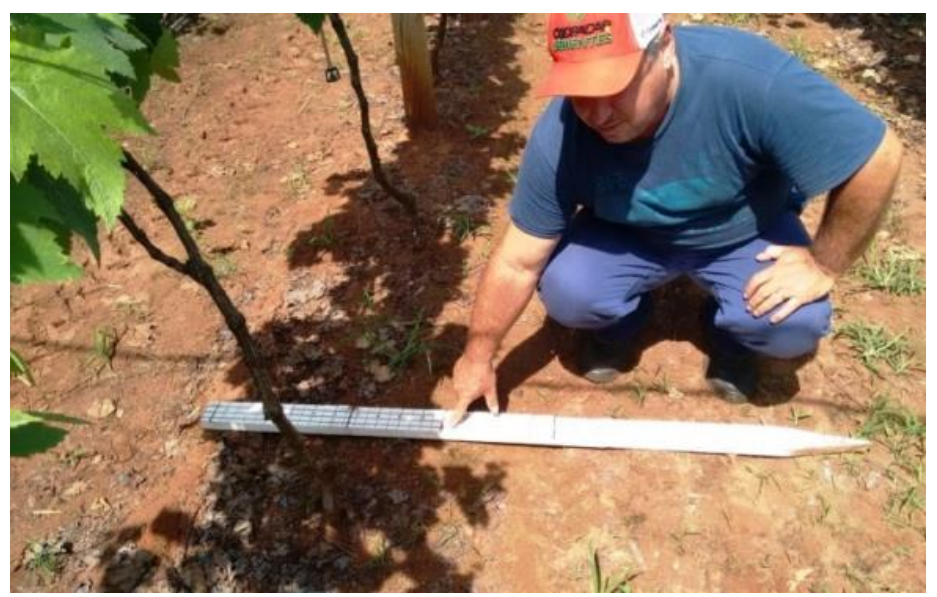

Figura 1. Uso de dispositivo para medir a largura da projeção da copa das videiras (LS). Votuporanga, SP.

\section{RESULTADOS E DISCUSSÃO}

Os porta-enxertos não influenciaram o desenvolvimento vegetativo da cultivar Isabel Precoce, uma vez que não foram observadas diferenças significativas quanto ao índice de área foliar (IAF), à altura das plantas (h) e à porcentagem da área sombreada pela copa (PAS) (Tabela 1). Essas são as variáveis mais empregadas, normalmente, na estimativa dos coeficientes de cultivo $(\mathrm{Kc})$ e da evapotranspiração da cultura, sob condições irrigadas (Williams \& Ayars, 2005; Allen \& Pereira, 2009; Hellman, 2016).

Tabela 1. Valores médios do índice de área foliar (IAF), da altura da copa (h), da porcentagem da área sombreada pelo dossel (PAS) para a cultivar Isabel Precoce sobre os porta-enxertos 'IAC 572' e 'IAC 766'. Votuporanga, 2015.

\begin{tabular}{cccc}
\hline Porta-enxerto & IAF $^{\text {ns }}$ & h (m) & PAS $\left.^{\mathbf{n s}} \mathbf{\%}\right)^{\mathbf{n s}}$ \\
\hline IAC 572 & 1,78 & 1,93 & 30 \\
IAC 766 & 1,99 & 1,99 & 31
\end{tabular}

${ }^{\text {ns }}$ Os tratamentos não apresentaram diferenças estatísticas pelo teste $\mathrm{F}$ ao nível de 5\% de probabilidade. 
Os valores de IAF foram superiores aos encontrados por Anzanelo \& Dutra de Souza (2015) e Sanchez-Rodriguez et al. (2016) para cultivares de Vitis labrusca conduzidas em espaldeira. Já as alturas da copa (h) são limitadas pela altura do aramado da espaldeira e podem variar conforme os fios forem posicionados. Os valores de PAS, por sua vez, apresentaram-se semelhantes aos obtidos por Montoro et al. (2017) e Moyer et al. (2015) para cultivares de Vitis Vinifera, também em espaldeira.

Verificou-se que a cultivar Isabel Precoce apresentou disponibilidade hídrica semelhante sobre os dois porta-enxertos (Figuras 2 e 3). Nas duas camadas monitoradas as tensões se situaram, em geral, dentro do intervalo entre $6 \mathrm{kPa}$ e $15 \mathrm{kPa}$, que são os limites recomendados para a região (Conceição, 2013). Os valores abaixo de $6 \mathrm{kPa}$ foram observados, principalmente, em períodos de maior precipitação pluvial, enquanto que os superiores a $15 \mathrm{kPa}$ foram registrados em apenas cinco dias, ao longo do ciclo da cultura.

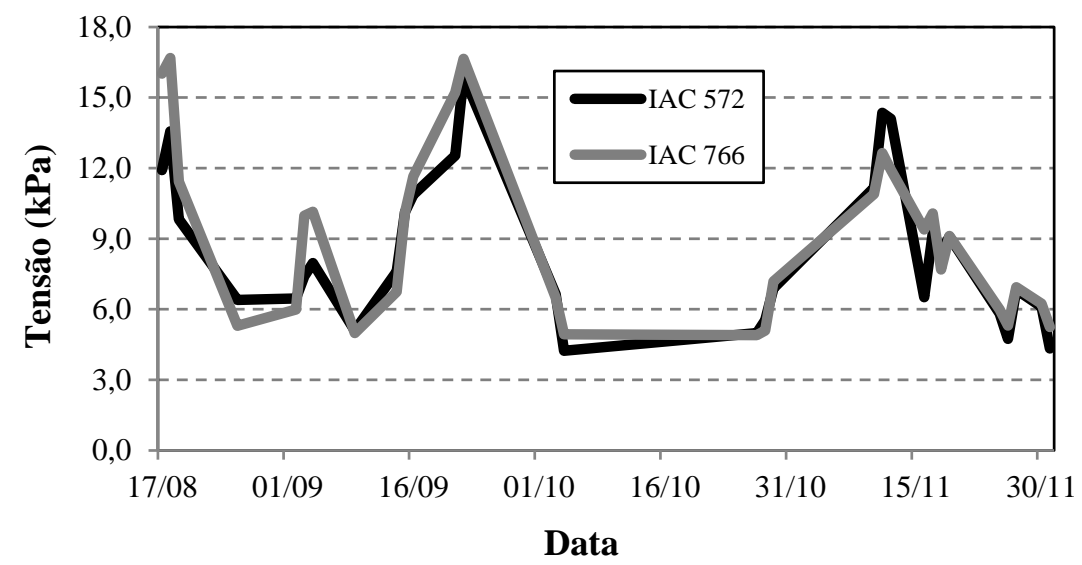

Figura 2. Tensões da água no solo na camada de 0 a $0,20 \mathrm{~m}$ de profundidade, para videiras 'Isabel Precoce' sobre portaenxertos 'IAC 572' e 'IAC 766'. Votuporanga, 2015.

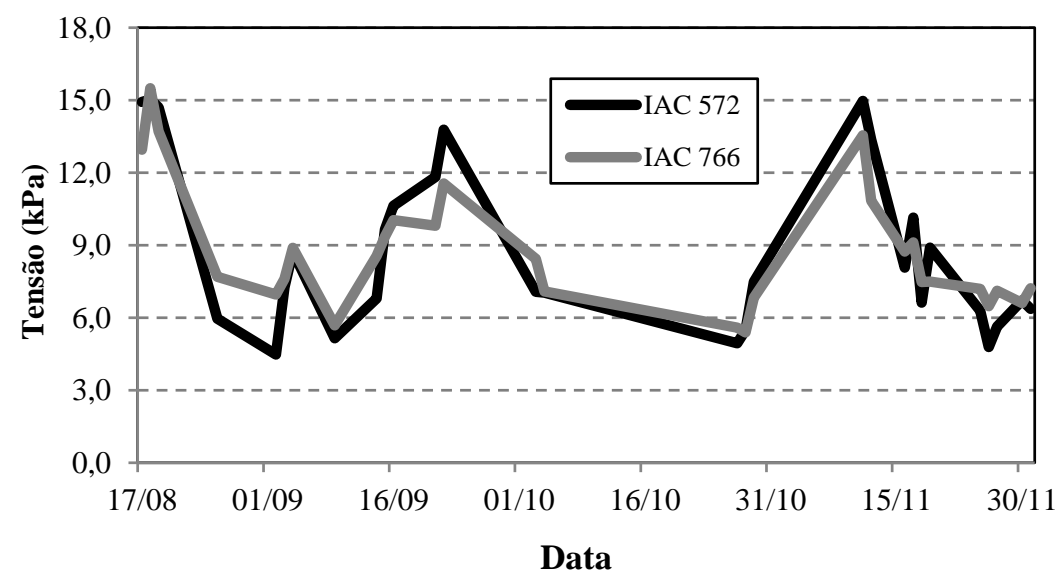

Figura 3. Tensões da água no solo na camada de $0,20 \mathrm{~m}$ a $0,40 \mathrm{~m}$ de profundidade, para videiras 'Isabel Precoce' sobre portaenxertos 'IAC 572' e 'IAC 766'. Votuporanga, 2015. 
Considerando-se os dados das duas camadas de solo conjuntamente, verifica-se que as tensões sob o porta-enxerto 'IAC 766' superestimaram as registradas com o 'IAC 572' em apenas $1 \%$ (Figura 4). Por outro lado, observa-se que houve uma dispersão maior, entre os valores, para as tensões mais elevadas. Nesse caso, os dois porta-enxertos tenderam a apresentar comportamento diferenciado refletindo, provavelmente, diferentes condutâncias estomáticas do dossel (Edwards et al., 2014). Assim, em estudos que envolvam avaliações sob condições de déficit hídrico, medidas de condutância estomática deverão ser incluídas.

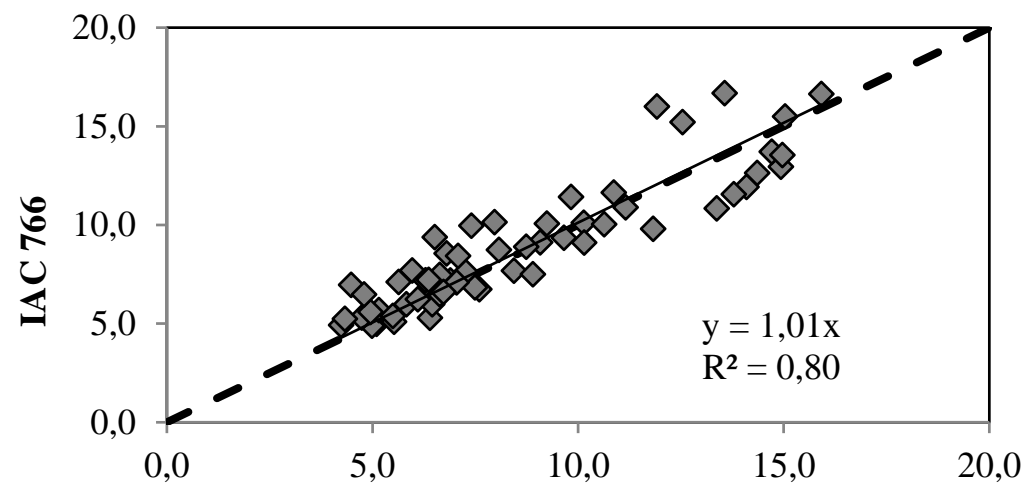

IAC 572

Figura 4. Tensões da água no solo $(\mathrm{kPa})$ na camada de 0 a $0,40 \mathrm{~m}$ de profundidade, para videiras 'Isabel Precoce' sobre portaenxertos 'IAC 572' e 'IAC 766'. Votuporanga, 2015.

\section{CONCLUSÃO}

Os porta-enxertos 'IAC 572' e 'IAC 766' não afetaram o desenvolvimento vegetativo e a disponibilidade de água no solo para a cultivar de videira de suco 'Isabel Precoce', em condições irrigadas.

\section{AGRADECIMENTO}

Os autores agradecem ao técnico Isaac Jesus de Souza, do Centro de Seringueira e Sistemas Agroflorestais do Instituto Agronômico de Campinas (IAC), pelo acompanhamento e sugestões apresentadas durante as avaliações. 


\section{REFERÊNCIAS}

ALEM, H.M.; GOUVÊA, L.R.L.; SILVA, G.A.P.; OLIVEIRA, A.L.B. de; GONÇALVES, P. de S. Avaliação de clones de seringueira para a região noroeste do Estado de São Paulo. Revista Ceres, v.62, n.5, p.430-437, 2015.

ANZANELlO, R.; DUTRA de SOUZA, P.V. Conteúdo de reservas, vigor vegetativo e rendimento de videiras submetidas a duas safras por ciclo vegetativo. Semina: Ciências Agrárias, v.36, n.2, p.719-729, 2015,

ALLEN, R. G.; PEREIRA, L. Estimating crop coefficients from fraction of ground cover and height. Irrigation Science, v. 28, p.17-34, 2009.

CAMARGO, U.A.; RITSCHEL, P.S.; MAIA, J.D.G. Variedades. IN: NACHTIGAL, J.C.; MAZZAROLO, A. Uva: o produtor pergunta, a Embrapa responde. Brasília: Embrapa Informação Tecnológica, 2008. p.25-31.

CAMARGO, U.A.; TONIETTO, J.; HOFFMANN, A. Progressos na viticultura brasileira. Revista Brasileira de Fruticultura, volume especial, p.144-149, 2011.

CONCEIÇÃO, M.A.F. Critérios para o manejo da irrigação de videiras em pequenas propriedades no noroeste paulista. Bento Gonçalves: Embrapa Uva e Vinho, 2013. 25p. (Embrapa Uva e Vinho. Circular Técnica, 98).

CONCEIÇÃO, M.A.F. Irrigação. IN: HOFFMANN, A.; SILVEIRA, S.V. da; GARRIDO, L. da R. (eds.). Produção integrada de uva para processamento: fertilidade e manejo do solo e da água. Brasília: Embrapa, 2015. p.29-39.

EDWARDS, E.J.; COLLINS, M.J.; BOETTCHER, A.; CLINGELEFFER, P.C.; WALKER, R.R. The role of rootstocks in grapevine water use efficiency: impacts on transpiration, stomatal control and yield efficiency. Acta Horticulturae, v.1038, p.121-128, 2014.

HELLMAN, E. Irrigation scheduling of grapevines with evapotranspiration data. AgriLIFE Extension, Texas A\&M University System. 6p. Disponível em: http://winegrapes.tamu.edu/files/2015/11/irrigationscheduling.pdf. Acesso em: 13 jun. 2016.

MONTORO, A.; LÓPEZ-URREA, R.; MAÑAS, F.; FERERES, E. Measures of transpiration in Vitis vinifera L. 'Tempranillo' in semi-arid climate. Acta Horticulturae, v.1157, p.209-214, 2017. 
MOYER, M.; PETERS, R. T.; HAMMAN, R. Conceptos básicos de riego para los viñedos del este de Washington. Pullman: Washington State University, 2015. 12p.

NOLZ, R.; CEPUDER, P.; BALAS, J.; LOISKANDL, W. Soil water monitoring in a vineyard and assessment of unsaturatedhydraulic parameters as thresholds for irrigation management. Agricultural Water Management, v.164, p.235-242, 2016.

REGINA, M.A.; PEREIRA, G.E.; CANÇADO, G.M.A.; RODRIGUES, D.J. Cálculo da área foliar em videira por método não destrutivo. Revista Brasileira de Fruticultura, v.22, n.3, p.310-313, 2000.

RIBEIRO, T.P.; LIMA, M.A.C de; ALVES, R.E. Maturação e qualidade de uvas para suco em condições tropicais, nos primeiros ciclos de produção. Pesquisa Agropecuária Brasileira, v.47, n.8, p.1057-1065, 2012.

SANCHEZ-RODRIGUEZ, L.A.; DIAS, C.T. dos S.; SPÓSITO, M.B. Fisiologia e produção da videira 'Niágara Rosada' nos sistemas de condução em espaldeira e em Y. Pesquisa Agropecuária Brasileira, v.51, n.12, p.1948-1956, 2016.

WILLIAMS, L.E.; AYARS, J.E. Grapevine water use and the crop coefficient are linear functions of the shaded area measured beneath the canopy. Agricultural and Forest Meteorology, v.132 p. 201-211, 2005. 